\title{
Impactos de la dinámica urbana en los mercados laborales en las principales ciudades chilenas*
}

\author{
Impacts of the urban dynamics \\ on labor markets in major Chilean cities
}

Luis Fuentes

Felipe Link

Felipe Valenzuela

\section{Resumen}

Las reformas de liberalización económica y financiera en la mayoría de los países de América Latina, provocaron un cambio radical en la base de producción material en la región. En este contexto, este trabajo pretende identificar y caracterizar el vínculo entre el crecimiento de las principales ciudades chilenas y la evolución de sus mercados laborales urbanos, buscando responder si estamos en presencia o no, de un proceso de urbanización de la estructura laboral. Específicamente, la hipótesis es que, a pesar de que las ciudades analizadas se distinguen en función de economías regionales especializadas, las estructuras de los diversos mercados laborales tienden a un proceso de homogenización, asociados a una dinámica eminentemente urbana y arrastrando con ello a la estructura de diferenciación social.

Palabras clave: urbanización; mercado de trabajo; estructura socio-territorial.

\begin{abstract}
Economic and financial liberalization reforms, in most Latin American countries, have led to a radical change in the structure of material production in the region. In this context, this paper aims to identify and characterize the link between the growth trends of the main Chilean cities and the evolution of their urban labor markets, seeking to clarify whether the labor structure has been undergoing a process of urbanization or not. Specifically, the hypothesis is that, although the analyzed cities are different regarding their specialized regional economies, the structures of the labor markets tend to undergo a process of homogenization, increasingly marked by urban dynamics and involving a process of internal differentiation of the urban social structure.
\end{abstract}

Keywords: urbanization; labor market; socialterritorial structure. 


\section{Introducción}

El conjunto de reformas de liberalización económica y financiera, que materializaron el "ajuste estructural" en la mayoría de los países de América Latina, provocaron un cambio radical en la base de producción material de los países de la región. A la reestructuración del mercado de trabajo y de organización de la producción, se sumó crecientemente una lógica financiera en la organización capitalista, lo que ha derivado en un nuevo estadio de modernización del sistema, que algunos autores denominan "régimen de acumulación financiarizado" (Chesnais, 2003). En este contexto, las ciudades pasan a ser lugares importantes para la movilización de capitales y específicamente, el sector inmobiliario se transforma en un actor representativo de esta fase de modernización capitalista. Desde el argumento de Lefebvre (1970), ya en los años setenta, se vislumbraba un cambio en esta dirección. En palabras del autor, se produce un cambio en la jerarquía de los circuitos de la acumulación, donde:

baja la parte de la plusvalía global formada y realizada en la industria, aumenta la parte de la plusvalía formada y realizada en la especulación y en la construcción inmobiliaria. El segundo circuito [inmobiliario] suplanta al principal [industrial]. De accidental, se transforma en principal. (Ibid., p. 212)

En un sentido similar, Harvey (2010, p. 178) señala que:

la reproducción del capitalismo implica la construcción de nuevas geografías, muchas veces mediante la destrucción creativa de las antiguas, una forma realmente expedita de afrontar el problema siempre presente del excedente de capital.

En la ciudad, este proceso toma la forma de las inversiones inmobiliarias con consecuencias tanto en la organización, funcionamiento, morfología y paisaje de las ciudades conectadas a la red urbana global (De Mattos, 2016), así como en las características del espacio social que se va configurando en estos lugares.

En este contexto general, este trabajo pretende identificar y caracterizar el vínculo entre las tendencias de crecimiento de las principales ciudades chilenas y la evolución de sus mercados laborales urbanos, buscando responder a las siguientes preguntas: ¿En qué medida los procesos de crecimiento de las ciudades inciden en una mayor importancia relativa de ciertas actividades económicas propiamente urbanas? ¿En qué medida ello puede tender hacia la homogenización de una estructura económica urbana crecientemente terciarizada? Es decir, si estamos en presencia o no, de un proceso de urbanización de la estructura laboral. Específicamente, la hipótesis que se pretende poner a prueba es que, a pesar de que las ciudades analizadas articulan economías regionales especializadas en distintas ramas de la actividad económica, las estructuras de los diversos mercados laborales urbanos tienden a un proceso de homogenización, arrastrando con ello a la estructura social urbana.

En la región existe una tradición de investigaciones que se han abocado a entender las transformaciones de los mercados de trabajo (Weller, 2011; OIT, 1998; Wormald 
y Ruiz Tagle, 1999; Kaztman, 2001). En particular, en Chile se han realizado estudios enfocados desde distintas perspectivas a entender los principales cambios transcurridos en el mundo del trabajo. El punto de partida de esta transformación tiene su origen en el "ajuste estructural" de mediados de los años 1970 en Chile, bajo la dictadura militar. Los cambios implementados reflejaron una política contestataria al modelo anterior y fuertemente marcada por la ideología neoliberal. El documento de base de la política económica de la dictadura militar chilena, denominado $E I$ ladrillo, postulaba entre otras cosas:

una rebaja en los costos de la mano de obra y mayor movilidad en el empleo; racionalización de los procesos de industrialización a favor del capital por sobre el trabajo, derogación de la ley de inamovilidad, modernización de los sistemas de subsidio de cesantía, reforma previsional, etc. (CEP, 1992, p. 190)

Es decir, además de reasignar y disminuir las funciones del Estado y utilizar indiscriminadamente al mercado para la asignación de recursos en diferentes áreas de la economía, la nueva política atacó directamente a la estructura del empleo y la producción. La idea predominante fue que

obreros y empleados han presionado por aumentos en sus ingresos reales más allá de los incrementos en productividad, en detrimento de las utilidades de las empresas y de los retornos al capital. (Ibid., p. 93)

Por lo tanto, la política propuesta tiene que ver con una significativa disminución del costo global del empleo, entendido como un inhibidor del crecimiento económico.
Así, uno de los ámbitos donde se expresaron con mayor fuerza las consecuencias del ajuste, fue en el mercado de trabajo, a través de la flexibilización. El largo proceso de reestructuración, apoyado y continuado después de 1990 por los gobiernos democráticos (Gárate, 2012) tuvo consecuencias en la transformación de la estructura socio ocupacional en Chile, donde disminuyó fuertemente la presencia de obreros, aumentaron considerablemente los trabajadores de servicios y se consolidaron los sectores dirigentes, principalmente en los centros urbanos, donde se concentró la actividad económica del país (De Mattos et al., 2005). En este contexto, siguiendo a Riffo (2003) existirían tres tendencias o ejes de discusión en torno a los efectos de la globalización y la reestructuración productiva en los mercados de trabajo, estos son: 1) el nuevo rol de las ciudades como lugares estratégicos para el capitalismo global; 2) el proceso de reestructuración de la base económica metropolitana asociada a la declinación de sectores dinámicos fordistas y el surgimiento de nuevos sectores dinámicos vinculados a los servicios avanzados; 3 ) la tendencia hacia nuevas formas de polarización social dentro de ciudades, particularmente en lo referente a la reestructuración de mercados de trabajo urbanos. Además, se observó una disminución de los niveles de sindicalización y de negociación colectiva (OIT, 1998) desempleo y expansión de relaciones laborales atípicas (Weller, 2011; Tokman, 2004), creciente pérdida de seguridad social (Wormald y Ruiz-Tagle, 1999), así como impactos en las identidades individuales y colectivas de los trabajadores principalmente 
del sector terciario (Diaz, Godoy y Stecher, 2006; Cárdenas, Link y Stillerman, 2010).

No obstante, mucho menor ha sido la evidencia para entender el vínculo de estos cambios en los contextos metropolitanos donde transcurren y se evidencian estas transformaciones (Lufin y Atienza, 2010; Ferrada y Zarzosa, 2010). Tal como lo señala Rubio (2002),

es bien sabido que el territorio con frecuencia es abordado como un continente prácticamente neutro de los fenómenos de carácter económico y social relacionados con el empleo y el trabajo. Por eso es preciso insistir en que el componente territorial es, en todo caso, un elemento diferenciador y condicionante de la estructura y dinámica de los mercados, ya que las características propias del modelo de desarrollo territorial tienen estrechas vinculaciones con ellos. (Rubio, 2002, p. 60).

En otras palabras, el autor devela la importancia de considerar al espacio metropolitano como una variable explicativa y vinculante de las transformaciones transcurridas en el mercado de trabajo, reconociendo las implicancias de estos cambios en el desarrollo territorial de las áreas metropolitanas.

Entre estos trabajos, es posible reconocer una convergencia en las transformaciones globales experimentadas por el mercado de trabajo, que en su esencia y comparado a décadas anteriores, representa un deterioro de las condiciones laborales, específicamente una mayor desprotección, precarización y flexibilización para la población económicamente activa de la región. Sin embargo, y en paralelo, también se reconoce una divergencia en términos de magnitudes e intensidades de estos procesos estructurales en cada uno de los mercados laborales locales urbanos. En otras palabras, estas investigaciones asumen que la evolución global del mercado de trabajo ha repercutido de distinta forma en los diferentes conglomerados urbanos, interrogándonos justamente sobre las particularidades, características y dinámicas que se expresarían territorialmente en mercados urbanos heterogéneos y desiguales, pero que responden a un proceso general de la dinámica urbana contemporánea.

Específicamente, la evolución territorial chilena reciente se ha caracterizado por un alto crecimiento de la urbanización, la cual hoy llega a casi el $90 \%$ de la población, con un aporte relevante de las ciudades intermedias. La actividad de la construcción ha mostrado un fuerte dinamismo, empujada por un creciente proceso de acceso al crédito hipotecario, una serie de modificaciones a los planes reguladores que han facilitado el crecimiento urbano tanto en extensión como en densificación y también por un incremento de los subsidios para vivienda otorgados por el Estado, los cuales se han ampliado socialmente hacia la clases medias.

Si en la década de los noventa, el principal cambio de las ciudades chilenas fue el paso de un país urbano a uno metropolitano (Hidalgo, De Mattos y Arenas, 2009), en la década más reciente podríamos decir que este fenómeno se ha ido consolidando y ha madurado.

Al respecto, este trabajo postula que tal patrón de crecimiento metropolitano que se inició en los noventa se ha consolidado pero también matizado, dada la manifestación de patrones de crecimiento urbano más 
complejos. Al mismo tiempo que las ciudades continúan expandiéndose, otros componentes del crecimiento urbano como la densificación, se han ido manifestado con fuerza en la configuración y trayectorias urbanas, generando nuevas geografías. Este proceso acelerado de desarrollo en algunas de estas ciudades ha sido activado principalmente por el rol que cumplen como articuladores de economías territoriales basadas en comodities vinculados a los recursos naturales y que han vivido un ciclo favorable en términos de su comercialización reciente en el mercado global. Todo esto está consolidando el proceso de metropolización de las ciudades regionales las cuales toman cada vez una mayor relevancia dentro del contexto nacional con lo cual se espraría que estas se diferenciaran más allá de las escalas, por su especificidad económico-laboral.

\section{Tendencias económicas e impacto en los mercados laborales}

El desarrollo socio-económico-político de las sociedades latinoamericanas en la segunda mitad del siglo XX, está marcado por el tránsito del modelo de Industrialización y Sustitución de Importaciones (ISI) al auge neoliberal. La implantación de las políticas de liberalización económica, se profundizaron en muchos casos con el proceso incipiente de globalización. Así, se comienza a gestar una respuesta al modelo de industrialización sustitutivo, a través de regímenes de acumulación extravertida, es decir, modelos de desarrollo centrados en la explotación de recursos naturales y la exportación de productos con bajo valor agregado en un contexto de globalización creciente. El caso de Chile es ejemplar en el contexto latinoamericano por la rapidez en que se implantó el modelo debido al régimen dictatorial represivo imperante.

Las consecuencias de esta revolución económica fueron, entre otras, una desarticulación casi completa de la capacidad industrial del país, con un altísimo costo social. Por su parte, las consecuencias en el plano social tienen que ver con una desregulación y precarización del trabajo, desmantelamiento de la organización sindical, privatización de empresas y reconversión laboral, etc. De este modo, una incipiente formación industrial es destruida orientando los esfuerzos hacia las exportaciones, con la consiguiente neo-taylorización en la organización de la producción y el aumento de la brecha en la distribución del ingreso (Moulian, 1997; Aquevedo, 1997).

Las consecuencias sociales de este proceso, son descritas por diferentes autores como un proceso contradictorio, que por una lado redujo el trabajo industrial y por otro, aumentó el trabajo precario, fragmentando aún más a una clase trabajadora heterogénea (Antunes, 1995). En las décadas siguientes, con la intensificación del proceso de globalización, la consolidación de las reformas neoliberales y la apertura cada vez mayor al mercado externo, los efectos sobre la estructura del empleo y el mercado laboral se profundizaron. No sólo en Chile y América Latina, sino también en los países centrales de Europa y en Estados Unidos, proliferaron los diagnósticos en torno a la transformación y precarización del 
empleo. Sassen (2001, p. 161) señala que: "los principales cambios en la organización de la actividad económica en los últimos quince años también han emergido como una fuente de inseguridad económica general $y$, particularmente, de nuevas formas de pobreza centradas en el empleo". De acuerdo con Sassen, pero en relación al caso de las ciudades latinoamericanas, Kaztman y Ribeiro (2008, p. 21) señalan también que:

una de las consecuencias de la ampliación de las fronteras de la competitividad, fue el aumento de la proporción de la población urbana para la cual el mundo del trabajo perdió estabilidad. Bajo el impulso de los procesos de desindustrialización, de reducción del Estado, así como de una acelerada incorporación de innovaciones tecnológicas en actividades muy distintas, disminuyó la proporción de ocupaciones protegidas y estables y crecieron las disparidades entre los ingresos y las tasas de desempleo y subempleo de trabajadores de alta y baja calificación.

Por su parte, De Mattos et al. (2005) constatan también un radical proceso de reestructuración económica con consecuencias de terciarización y desindustrialización, donde a pesar de observar una ligera reducción de la regresividad en la distribución del ingreso, así como una significativa reducción de la pobreza y de la indigencia, señalan la persistencia de una situación de acentuadas desigualdades sociales (Link, 2010).

Ahora, específicamente el mercado de trabajo chileno presenta actualmente una serie de características donde destacan el alto desempleo juvenil, la baja participación femenina, la alta rotación laboral, la temporalidad de los contratos de trabajo, la inseguridad en el empleo y las dificultades en la inserción laboral, especialmente de jóvenes y mujeres.

Así, el nuevo contexto modifica la relación tradicional entre capital y trabajo del fordismo, generando inseguridad y eventualmente exclusión. Por lo tanto,

dentro de las políticas para combatir la exclusión, el empleo ocupa un rol central, porque contiene elementos integrativos fundamentales, como son, los ingresos, identidad social, legitimidad y reconocimiento social, contactos e integración a redes, participación en acciones colectivas, etc. (Ruiz-Tagle, 2000, p. 17)

La reestructuración de la economía, en la producción y el trabajo, tuvo profundas consecuencias también, sobre la forma que asume la estratificación social. Para el caso chileno, León y Martínez (2007) señalan un tipo de desigualdad, como resultado de la reestructuración, con consecuencias de largo plazo, más allá de las obvias diferencias cuantitativas en las capacidades de consumo presente. Para estos autores surgen: "barreras a la movilidad que dan origen a conjuntos típicamente diferenciados, por razones adscriptivas, no solo en sus probabilidades de reproducción, sino también en estilos de vida y ethos cultural" (León y Martínez, 2007, p. 303). Es decir, que el impacto de la reestructuración en Chile bajo los efectos de la globalización afecta no sólo las características socioeconómicas de la población, sino que afecta también, a la forma de la estratificación social basada en el trabajo. La matriz de categorías sociales de León y Martínez (2007) va más allá de las meras construcciones analíticas de estratos 
socioeconómicos, basados en variables continuas como el ingreso o la educación. Las conclusiones de estos autores tienen que ver con: a) un distanciamiento de la clase obrera de los sectores medios; b) un mejoramiento de los ingresos laborales de las categorías de trabajadores independientes en comparación con las posiciones asalariadas; c) un cambio en la composición social de la pobreza; lo que provoca un distanciamiento interclasista con movilidad social horizontal y no vertical, que impide el salto de un estamento a otro (León y Martínez, 2007). Las consecuencias de estos procesos en la ciudad, tienen que ver con la marginalización y el aislamiento social de los pobres urbanos (Kaztman, 2001).

En la misma línea de análisis de la evolución en la estratificación social chilena, Torche y Wormald (2007, p. 339) señalan que

el paso de un modelo sustitutivo de importaciones, liderado por el Estado, a otro abierto, basado en la competencia en el mercado y liderado por los privados, afectaron la estructura social y las oportunidades de vida de las personas provocando marcados contrastes en la sociedad.

Basados en una adaptación del esquema de clases de Goldthorpe, Torche y Wormald (2007) concluyen que la estructura ocupacional chilena:

ha tendido a reproducir relaciones de servicios y contractuales en ocupaciones definidas como formales, traduciéndose en el fortalecimiento de la clase de servicio y de los segmentos asalariados 0 dependientes en ocupaciones calificadas y no calificadas (...) se ha expandido la clase de los pequeños empresarios acorde con la reducción de las ocupaciones en el aparato estatal (...) debilitando el componente clasista en la nueva estructura. (Torche y Wormald, 2007, p. 383).

Sin embargo, a pesar de lo anterior y de la misma manera que en el estudio de León y Martínez (2007), se constata que si bien la movilidad total es significativa, dada la reestructuración, ésta es de corta distancia y no implica un asenso significativo en el estatus socioeconómico de las personas. Es decir, que los impactos de la reestructuración tienen consecuencias importantes sobre la movilidad y las posibilidades de ascenso social.

\section{Tendencias recientes del desarrollo urbano en Chile}

En cuanto al desarrollo urbano, sucedió algo similar al proceso de reestructuración productiva. Según Daher (1995), en Chile el modelo liberal ortodoxo de fines de los setenta se tradujo en la formulación en 1979, de una nueva Política Nacional de Desarrollo Urbano. Esta política, en una de sus más características aseveraciones, expresó: "el suelo urbano no es un recurso escaso", "Se definirán procedimientos y se eliminarán restricciones de modo de permitir el crecimiento natural de las áreas urbanas, siguiendo las tendencias del mercado" (Minvu, 1981, pp. 10-13 en Daher, 1995). Respecto de la vivienda, la política indicaba que "el Estado fomentará y apoyará la creación de un mercado abierto de vivienda. La responsabilidad de producción corresponde al sector privado" (Minvu, 1981, p. 14 en Daher, 1995). Siguiendo a Sabatini (2000, p. 49): 
la temprana y radical liberalización de los mercados urbanos chilenos tuvo consecuencias inesperadas en los precios del suelo y en la segregación residencial en Santiago (...) Se trata de dos fenómenos de relevancia para el desarrollo urbano, en general, y que tienen un impacto directo en el grave problema de la pobreza urbana, en particular: los precios del suelo condicionan el acceso de los pobres al suelo; y el patrón de segregación residencial, sus perspectivas de integración social.

Específicamente, la erradicación forzada de pobladores a zonas periféricas de la ciudad (Rojas, 1984), así como la posterior implementación de la política de subsidio habitacional dependiente de los precios en el mercado de suelo, fueron configurando ciudades fragmentadas y segmentadas, tanto en la estructura social como en el territorio. Por lo tanto, el correlato espacial de la transformación económica en la producción y el trabajo, se presenta en términos de fragmentación, como una imagen de ciudad diferente al modelo de centro y periferia y bajo el supuesto que "lo que debía mostrar un funcionamiento global, estalló en múltiples unidades y que no habría ya una unidad del conjunto urbano" (PrevotShapira, 2001, p. 38).

Así, la evolución territorial chilena se ha caracterizado por un alto crecimiento de la urbanización, la cual hoy llega a casi el $90 \%$ de la población, con un aporte relevante de las ciudades intermedias. La actividad de la construcción ha mostrado un fuerte dinamismo, empujada por un fuerte proceso de acceso al crédito hipotecario de la población, una serie de modificaciones a los planes reguladores que han facilitado el crecimiento urbano tanto en extensión como en densificación y también por un fuerte incremento de los subsidios para vivienda otorgados por el Estado, los cuales se han ampliado socialmente hacia la clases medias. Así, los últimos datos de crecimiento demográfico y de vivienda muestran que el crecimiento ha estado fuertemente concentrado en las ciudades del norte de Chile, que articulan economías regionales especializadas en la extracción de materias primas, principalmente minerales (cobre) y también en una ciudad del sur del país (Puerto Montt), ciudad capital de una región especializada en la producción de salmón para exportación (ver Gráfico 1).

En estas ciudades, el crecimiento de las viviendas ha sido muy importante. Así por ejemplo, el caso de Puerto Montt concentra prácticamente el $70 \%$ de la edificación residencial en los últimos veinte años, como se observa en el Gráfico 2. Las ciudades del norte de Chile también presentan cifras muy altas superando el $50 \%$.

Si bien, el análisis realizado no permite detallar los patrones específicos de segregación de cada una de las 24 ciudades, sí podemos afirmar la consolidación de una dinámica urbana que se ha intensificado sobre todo en los últimos veinte años. Como se observa en el Gráfico 2, a través del crecimiento porcentual de los predios habitacionales, entendido como un indicador eficiente para caracterizar este proceso. Algunos estudios recientes, dan cuenta de la especificidad de ciudades particulares en vías de metropolización (Hidalgo, De Mattos y Arenas, 2009), sin embargo, el objetivo de este trabajo es conocer una tendencia general, a partir del análisis conjunto de una muestra importante de la diversidad urbana en Chile. 


\section{Gráfico 1 - Tasa de crecimiento anual de población 1992-2012}

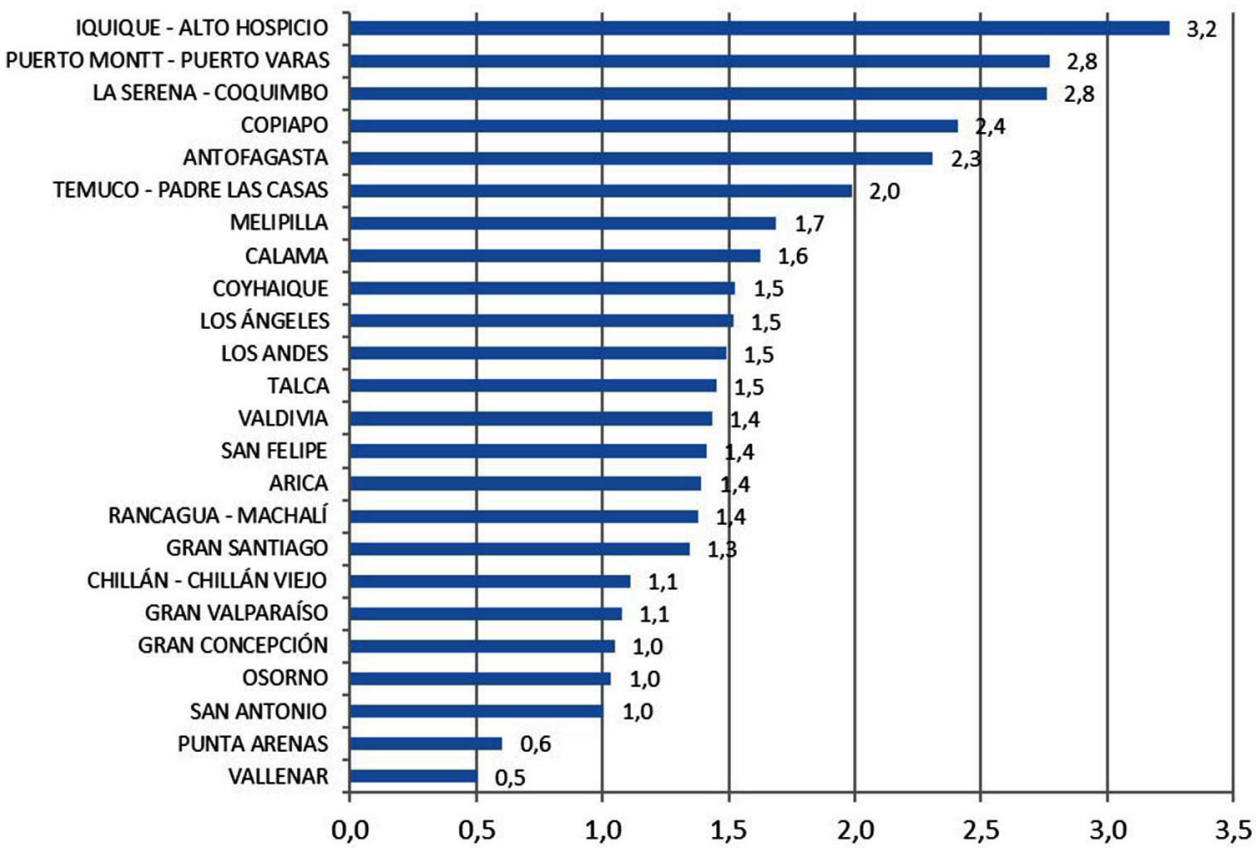

Fuente: Elaboración propia en base a información censal.

\section{Gráfico 2 - Porcentaje de predios habitacionales construidos 1990-2010}

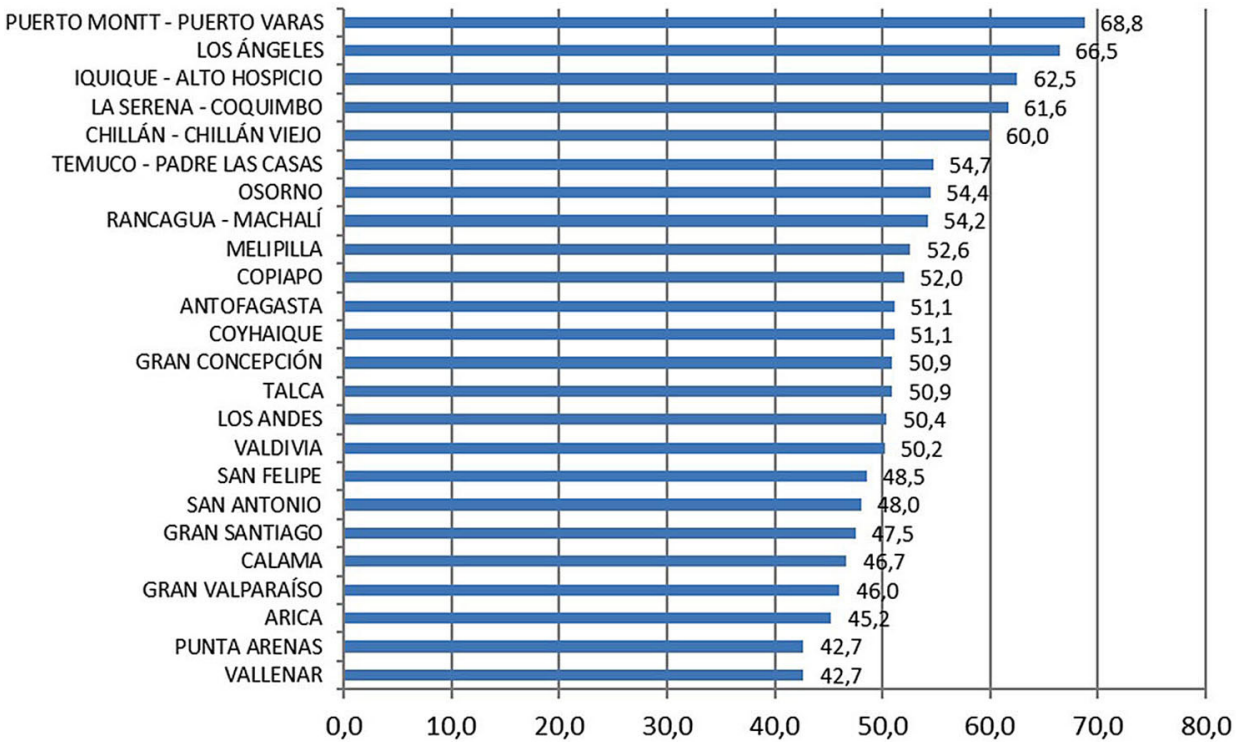

Fuente: Elaboración propia en base a información Servicio de Impuestos Internos. 


\section{Identificando agrupaciones de mercados laborales urbanos en Chile}

Con el objetivo de caracterizar la estructura de los mercados laborales en las principales ciudades chilenas, y conocer cuáles son las semejanzas y diferencias entre ellas en el contexto de sus variadas economías locales, a la luz de la reestructuración, se generaron tipologías de ciudades en función de las actividades económicas donde se emplea la población. De esta forma, fue posible identificar los distintos tipos de estructura laboral existentes en las ciudades chilenas, permitiendo hacer comparaciones entre ellos y realizar un análisis de su evolución en el tiempo.

Para realizar esto, se seleccionaron 24 ciudades chilenas (ver Mapa 1) donde se realizó un análisis de las actividades económicas de la población ocupada, utilizando los datos provenientes de la Encuesta Casen 1992 y 2011. De esta forma, fue posible dar cuenta de las transformaciones de la estructura de los mercados laborales durante las últimas dos décadas. Las actividades económicas, por su parte, fueron caracterizadas a través de la Clasificación Internacional Industrial Uniforme de 1976 (División Estadística de Naciones Unidas, n.d.), que permite una amplia desagregación.

Durante el período analizado, las principales tendencias a nivel agregado tienen que ver con el crecimiento en la importancia relativa de las actividades terciarias a costa principalmente de las actividades secundarias, pues mientras las primeras crecen en casi 10 puntos porcentuales entre 1992 y 2011, el porcentaje de población empleada en el sector secundario disminuye de $30,2 \%$ a 21,6\% (ver Gráfico 3).

\section{Mapa 1 - Ciudades chilenas seleccionadas}

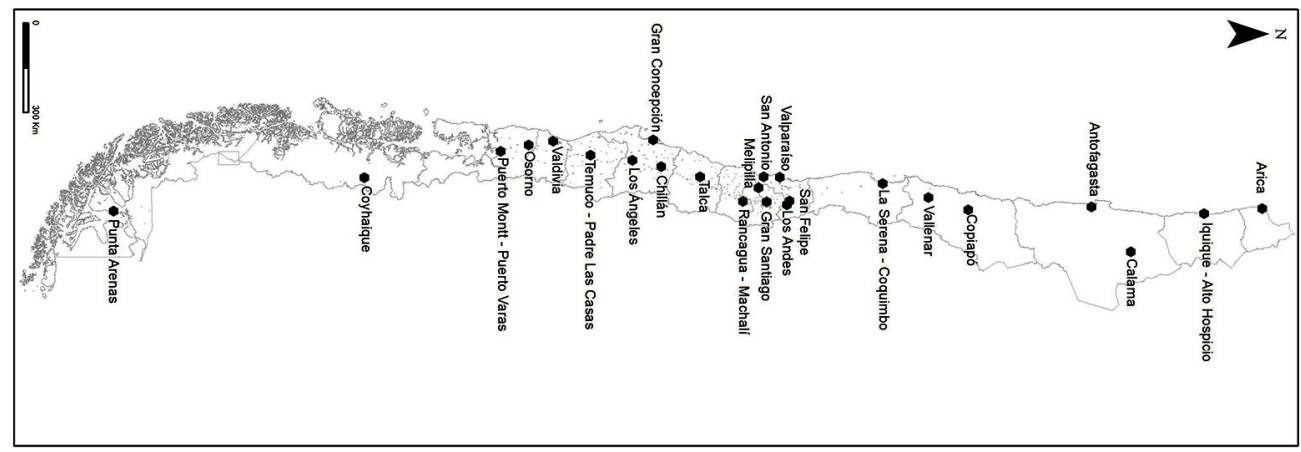


Gráfico 3 - Distribución por sector de la actividad económica en que trabajan los ocupados de las 24 ciudades analizadas

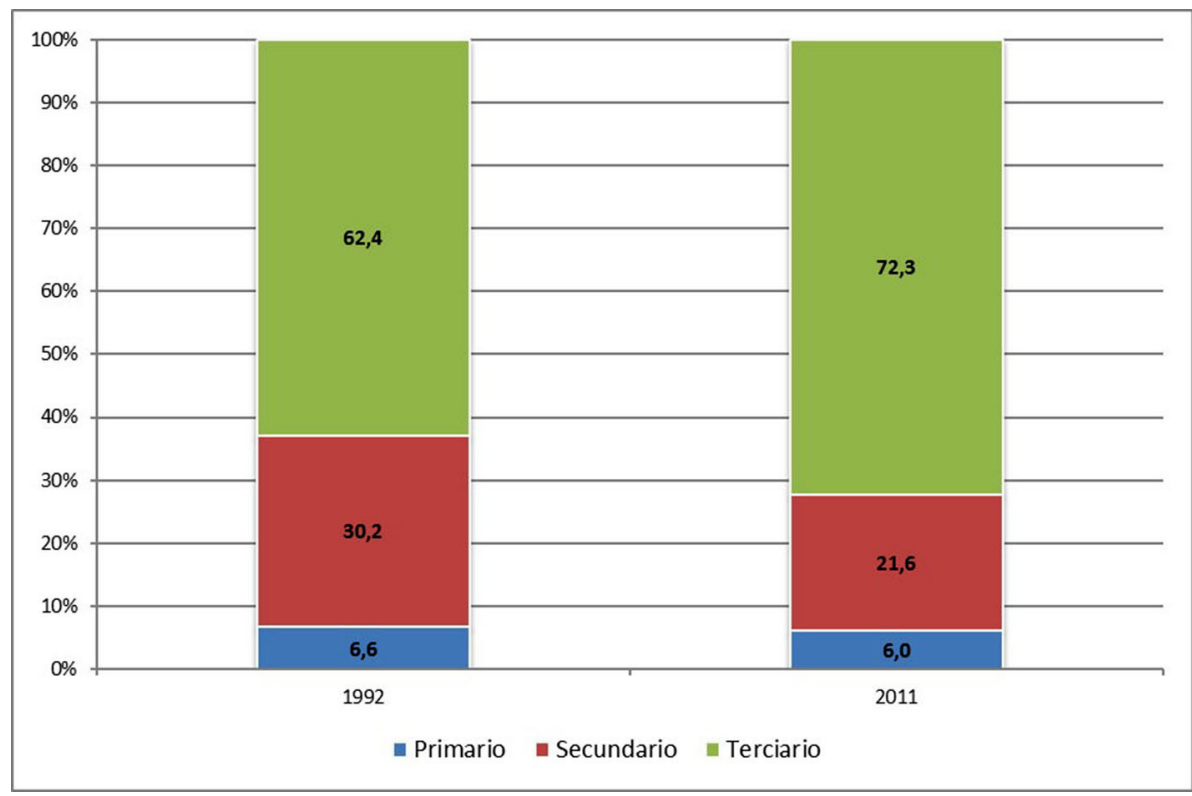

Analizando las actividades económicas a un nivel más específico, se observa que la mayoría de los rubros de mayor relevancia son los relacionados al comercio y los servicios, como el comercio al por menor, la administración pública y defensa, los servicios personales y de los hogares, y los servicios sociales (ver Gráfico 4). Entre ellos, el comercio, la administración pública y defensa, y los servicios de saneamiento son los que presentan crecimientos más significativos en el período. Mientras tanto, es destacable que pese a la disminución en la importancia relativa del sector secundario, el rubro de la construcción continúa siendo muy importante en las ciudades analizadas e incluso ha crecido su participación relativa en el empleo. Es así como al año 2011, más del 10\% de la población de estas ciudades trabajaba en el sector de la construcción.

Posteriormente, a partir de la composición de los mercados laborales urbanos de cada ciudad según actividad económica, se realizó un Análisis Factorial de Correspondencias Simples (AFC) con los datos de cada año analizado (1992 y 2011). Este procedimiento estadístico tiene como objetivo explicar la estructura de las covariaciones entre variables, mediante la definición de una cierta cantidad de factores. Este método se aplica para descubrir dimensiones latentes y una estructura subyacente a los datos, que permitan interpretar manifestaciones visibles (Buzai, 2003). En este caso, se buscó 
Gráfico 4 - Porcentaje de población ocupada

en los 10 sectores económicos de mayor importancia

en las 24 ciudades seleccionadas (1992-2011)

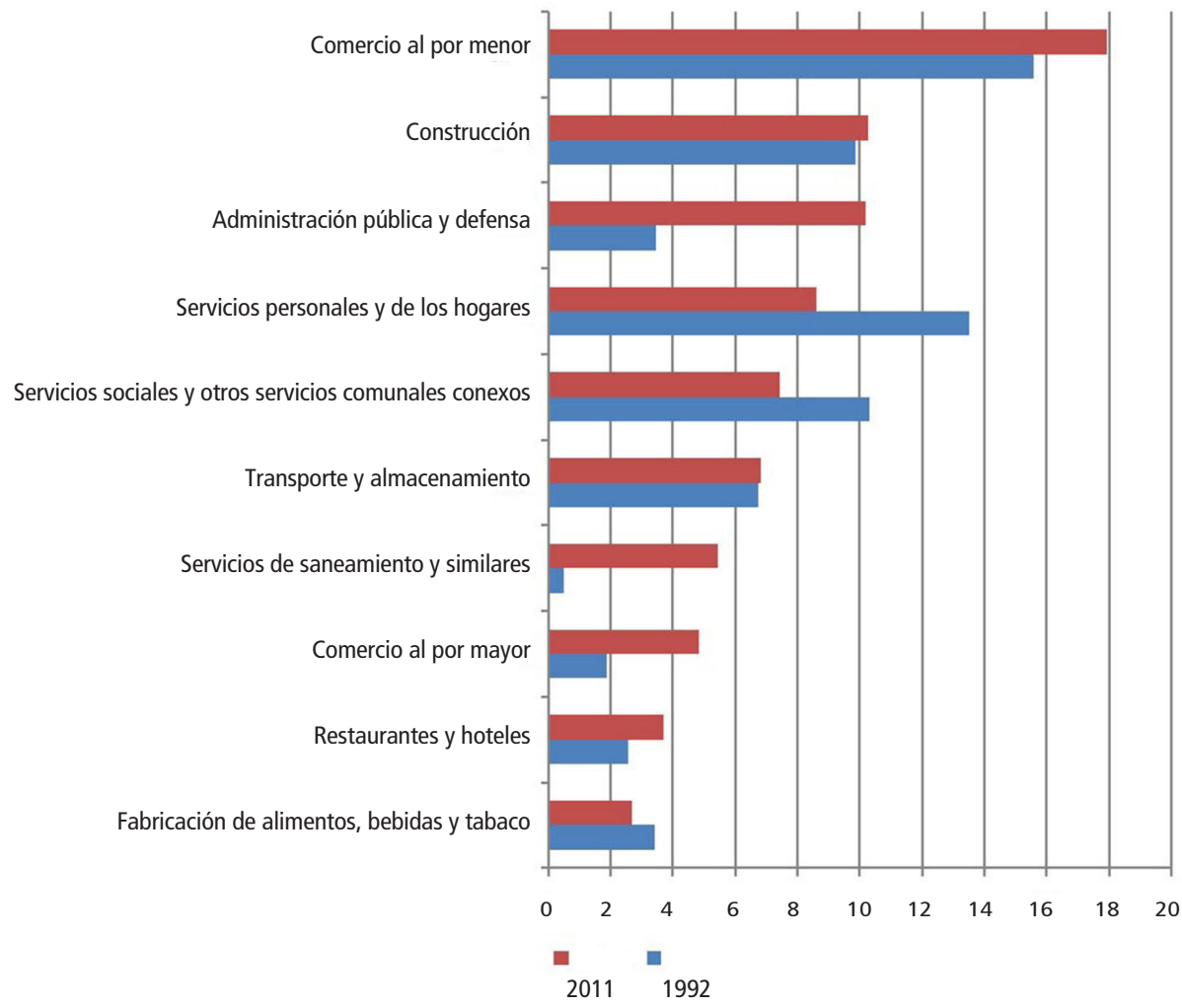

conocer cuál es la estructura subyacente en la composición de los mercados laborales urbanos en las 24 ciudades analizadas, con el objetivo de identificar patrones de diferenciación. Una vez realizado el AFC, se generaron las tipologías (o clúster) a través de un método de clasificación jerárquica, que permite obtener grupos internamente homogéneos, y heterogéneos entre sí.
A través de este análisis, tanto en 1992 como en 2011 el resultado de agrupación óptimo generado por el proceso de clasificación jerárquica, indicó la conformación de seis clúster o grupos de ciudades. En el Cuadro 1 se muestran las ciudades que componen estos clúster en ambos años, lo que permite advertir desde ya que se producen algunos cambios relevantes a lo largo del período. Una 
Cuadro 1 - Clasificación de las ciudades analizadas por clúster

\begin{tabular}{|c|c|c|c|c|c|c|}
\hline & \multicolumn{6}{|c|}{ Clúster } \\
\hline & Metropolitano & Primario-minero & $\begin{array}{l}\text { Portuario- } \\
\text { pesquero }\end{array}$ & Primario-agrícola & Energético & $\begin{array}{l}\text { Intermedias } \\
\text { terciatizadas }\end{array}$ \\
\hline 1992 & $\begin{array}{l}\text { Gran } \\
\text { Santiago }\end{array}$ & $\begin{array}{l}\text { Calama } \\
\text { Copiapó } \\
\text { Vallenar } \\
\text { Los Andes } \\
\text { Rancagua-Machalí }\end{array}$ & $\begin{array}{l}\text { Iquique-Alto } \\
\text { Hospicio } \\
\text { San Antonio } \\
\text { Gran Concepción } \\
\text { Puerto Montt- } \\
\text { Puerto Varas }\end{array}$ & $\begin{array}{l}\text { San Felipe } \\
\text { Melipilla } \\
\text { Los Ángeles } \\
\text { Chillán-Chillán } \\
\text { Viejo } \\
\text { Osorno }\end{array}$ & Punta Arenas & $\begin{array}{l}\text { Arica } \\
\text { Antofagasta } \\
\text { La Serena-Coquimbo } \\
\text { Gran Valparaíso } \\
\text { Talca } \\
\text { Temuco-Padre las Casas } \\
\text { Valdivia } \\
\text { Coyhaique }\end{array}$ \\
\hline 2011 & $\begin{array}{l}\text { Gran } \\
\text { Santiago } \\
\text { Gran } \\
\text { Valparaíso }\end{array}$ & $\begin{array}{l}\text { Calama } \\
\text { Copiapó } \\
\text { Vallenar } \\
\text { Los Andes } \\
\text { Rancagua-Machalí } \\
\text { Antofagasta }\end{array}$ & $\begin{array}{l}\text { Puerto Montt- } \\
\text { Puerto Varas }\end{array}$ & $\begin{array}{l}\text { San Felipe } \\
\text { Melipilla }\end{array}$ & Punta Arenas & $\begin{array}{l}\text { Arica } \\
\text { La Serena-Coquimbo } \\
\text { Talca } \\
\text { Temuco-Padre las Casas } \\
\text { Valdivia } \\
\text { Coyhaique } \\
\text { Iquique-Alto Hospicio } \\
\text { Gran Concepción } \\
\text { Chillán-Chillán Viejo } \\
\text { Osorno } \\
\text { San Antonio } \\
\text { Los Ángeles }\end{array}$ \\
\hline
\end{tabular}

vez identificados estos clúster, se realiza una caracterización en función de sus actividades económicas predominantes y de aquellas que los diferencian respecto a los otros clúster. Este análisis permite asignarle los nombres que aparecen en el Cuadro 1.
A continuación se analizan los cambios en la conformación de los clústers entre 1992 y 2011, buscando estudiar en qué medida se está produciendo una homogenización de los mercados laborales urbanos. 


\section{La evolución de los clústers de mercados laborales entre 1992 y 2011 : de la especialización a la homogenización}

Para realizar el análisis de la composición y evolución de los clústers se consideraron tres factores principales:

1) Las actividades predominantes en cada clúster, es decir, aquellas actividades donde se desempeña un mayor porcentaje de la población. Cabe señalar que debido a la relevancia transversal de sectores como el comercio y la construcción, los clústers no se diferencian mayormente entre sí en este sentido.

2) Las actividades distintivas de cada clúster, es decir, aquellas que en mayor medida lo diferencian del resto de los clúster. Estas actividades son las que obtienen un mayor "valor test" en la conformación de las tipologías. El valor test de una actividad resulta más alto en la medida en que el aporte del clúster al total general de esa actividad es superior a la proporción que representa el clúster respecto al total de ocupados. Además, el valor test tendrá a ser más alto en la medida en que la actividad en cuestión tenga una mayor importancia relativa dentro del clúster.

3) Los cambios en las ciudades que conforman los clústers, considerando las ciudades que dejan de pertenecer y las que se suman a cada uno de ellos.

\section{Clúster metropolitano}

El primer clúster es denominado metropolitano, ya que está conformado por ciudades de esa escala y por lo tanto se caracteriza por concentrar actividades de carácter metropolitano. En 1992 el clúster se conformaba sólo por el Gran Santiago, y se distinguía principalmente por ciertas actividades industriales, como la fabricación de productos textiles, químicos y metálicos. Durante las décadas posteriores, la actividad industrial fue perdiendo peso, y el significado de la centralidad metropolitana se traslada progresivamente desde la actividad industrial hacia los servicios, destacando los Servicios personales y de los hogares y las Comunicaciones, como muestran los resultados del 2011. En este escenario, el Gran Valparaíso se diferencia de otras ciudades de tamaño intermedio y adquiere también un carácter metropolitano, asemejándose más al Gran Santiago en términos de sus actividades económicas, por lo que pasa a formar parte de este clúster.

\section{Clúster primario-minero}

El clúster minero es el que presenta un mayor nivel de especialización y por lo tanto es probablemente el de más fácil identificación, debido a la gran relevancia que tiene la actividad minera en las ciudades que lo componen. Esta característica se ha mantenido entre 1992 y 2011, observándose incluso un crecimiento en la importancia 
relativa de la minería en el empleo de estas ciudades, que pasa de un $16,9 \%$ a un $18,3 \%$. La economía de estas ciudades está basada fuertemente en la minería, por lo que en buena medida el resto de las actividades económicas dependen o son complementarias a ella. El año 1992 este clúster se componía de las ciudades de Calama, Copiapó, Vallenar, Los Andes y Rancagua-Machalí, a las que se sumó Antofagasta en 2011.

\section{Clúster portuario-pesquero}

Este clúster es uno de los que ha experimentado mayores cambios entre 1992 y 2011. Hacia 1992, se componía de varias ciudades costeras con una alta importancia de las actividades portuarias (expresadas en la rama de transporte y almacenamiento) y pesqueras. Sin embargo, dos décadas después, estas actividades perdieron importancia relativa en los mercados laborales de algunas ciudades que pasaron a formar parte del amplio grupo que denominamos "ciudades intermedias terciarizadas": Iquique-Alto Hospicio, San Antonio y el Gran Concepción corresponden a ese grupo. De esta forma, en 2011 este clúster está conformado exclusivamente por Puerto Montt-Puerto Varas, que muestra una alta especialización en Pesca, concentrando el $37,5 \%$ de los ocupados en esta actividad en el total de las 24 ciudades analizadas. Al mismo tiempo, actividades como la agricultura y el turismo pasan a ser distintivas también de este clúster.

\section{Clúster primario-agrícola}

El clúster agrícola agrupa a las ciudades especializadas en actividades primarias agropecuarias. Hacia 1992 este clúster se componía de varias ciudades intermedias: San Felipe, Melipilla, Los Ángeles, Chillán y Osorno. Sin embargo, debido a la pérdida en la importancia relativa de este tipo de actividad frente al crecimiento de las actividades terciarias, en 2011 sólo San Felipe y Melipilla mantienen su nivel de especialización agrícola y son las únicas que conforman el clúster, mientras las restantes pasan a formar parte del grupo de intermedias terciarizadas. Las actividades de agricultura y caza son la fuente de empleo del $21,6 \%$ de los trabajadores del clúster en 2011, lo que demuestra su nivel de especialización en relación al total de las ciudades analizadas, donde ese porcentaje alcanza sólo el 2,3\%.

\section{Clúster energético}

Este clúster se compone exclusivamente por la ciudad de Punta Arenas y se ha mantenido constante entre 1992 y 2011. Si bien esta ciudad tiene una composición de su economía bastante similar a la de otras ciudades, se destaca fundamentalmente por la existencia de ciertas actividades que, si bien no tienen una importancia muy grande en la estructura del empleo local, se realizan muy poco en otras ciudades del país. La principal de estas actividades es la producción de petróleo 
crudo y gas natural, que hacia 1992 estaba concentrada en un $83,4 \%$ en Punta Arenas. Este porcentaje disminuyó en 2011 (58,1\%), pero se le sumó una especialización en la extracción de carbón, en la que Punta Arenas concentra al $56,9 \%$ de los ocupados totales.

\section{Ciudades intermedias terciarizadas}

El último clúster corresponde a un conjunto de ciudades intermedias que se caracterizan por tener un alto porcentaje de sus ocupados en actividades terciarias y un bajo porcentaje en actividades primarias, pese a que algunas de ellas aparecen entre sus actividades distintivas. Esto significa que se trata de ciudades que no tienen una especialización clara en actividades ligadas a la extracción de recursos naturales, como ocurre principalmente con los clúster minero o agrícola, pero tampoco alcanzan una complejidad suficiente como para asemejarse a las ciudades metropolitanas. En 1992 este clúster estaba conformado por las ciudades de Arica, Antofagasta, La Serena-Coquimbo, Gran Valparaíso, Talca, Temuco-Padre las Casas, Valdivia y Coyhaique. Dos décadas después, la mitad de las ciudades analizadas quedan clasificadas en este clúster, agregándose Iquique-Alto Hospicio, San Antonio, Chillán, Gran Concepción, Los Ángeles y Osorno. Estas ciudades tienden a perder el nivel de especialización que las hacía conformar parte de otro clúster, y tienden a parecerse más entre sí como parte de un proceso de homogenización que se caracteriza por la preponderancia de las actividades terciarias propiamente urbanas, avanzando hacia la metropolización desde el punto de vista de la estructura del empleo.
En el siguiente cuadro se observa las actividades distintivas y las actividades predominantes para cada cluster en cada año analizado. Es especialmente interesante analizar el cambio en las actividades distintivas. En 1992 en general las tres actividades que caracterizan el cluster estaban vinculadas a su particularidad productiva (pesca, minería, energía, entre otros). Sin embargo hacia el 2011 esto varía y se van incorporando actividades terciarias, la mayoría relacionadas al proceso de urbanización generalizado del país. Estas actividades tienen una fuerte presencia en todas las ciudades, lo interesante aquí es que pasan a ser distintivas de la estructura del mercado laboral urbano.

\section{Consideraciones finales}

Finalmente, a partir del análisis de las 24 ciudades en Chile y la identificación de estos grupos de ciudades y su evolución en las últimas dos décadas, es posible caracterizar los procesos de transformación de los mercados urbanos del trabajo, en un contexto de fuerte crecimiento demográfico y económico. En este sentido, ante la pregunta por un proceso de urbanización de la estructura laboral, la principal tendencia que se puede identificar es hacia la homogenización de la base económica de muchas ciudades intermedias, donde las actividades terciarias vinculadas al comercio y los servicios propiamente urbanos aumentan su protagonismo en la estructura del empleo. Ello se ve reflejado en el crecimiento del clúster que denominamos como "ciudades intermedias terciarizadas". Como parte de este mismo proceso, varias ciudades 


\section{Cuadro 2 - Actividades económicas distintivas y predominantes por clúster de ciudades}

\begin{tabular}{|c|c|c|c|c|c|c|}
\hline \multirow{3}{*}{ 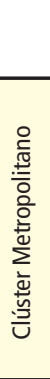 } & \multirow[b]{2}{*}{1992} & \multicolumn{2}{|l|}{$\begin{array}{l}\text { Actividades distintivas } \\
\text { (\% dentro del clúster) }\end{array}$} & \multicolumn{2}{|l|}{$\begin{array}{l}\text { Actividades predominantes } \\
\text { (\% respecto al total de todos los clúster) }\end{array}$} & \multirow{2}{*}{$\begin{array}{r}\text { Ciudades } \\
\text { Gran Santiago }\end{array}$} \\
\hline & & $\begin{array}{l}\text { Comercio al por menor } \\
\text { Servicios personales y de los hogares } \\
\text { Construcción }\end{array}$ & $\begin{array}{l}15,3 \\
14,8 \\
9,8\end{array}$ & $\begin{array}{l}\text { Textiles, prendas de vestir e industria del cuero } \\
\text { Fabricación de (...) derivados del petróleo, } \\
\text { carbón, caucho y plástico } \\
\text { Fabricación de productos metálicos, } \\
\text { maquinaria y equipo }\end{array}$ & $\begin{array}{l}79,4 \\
82,7 \\
75,1\end{array}$ & \\
\hline & 2011 & $\begin{array}{l}\text { Comercio al por menor } \\
\text { Administración pública y defensa } \\
\text { Construcción }\end{array}$ & $\begin{array}{l}18,9 \\
11,1 \\
10,1\end{array}$ & $\begin{array}{l}\text { Servicios personales y de los hogares } \\
\text { Fabricación de (...) derivados del petróleo, } \\
\text { carbón, caucho y plástico } \\
\text { Comunicaciones }\end{array}$ & $\begin{array}{l}69,7 \\
83,0 \\
77,8\end{array}$ & $\begin{array}{l}\text { Gran Santiago } \\
\text { Gran Valparaíso }\end{array}$ \\
\hline \multirow{2}{*}{ } & 1992 & $\begin{array}{l}\text { Comercio al por menor } \\
\text { Extracción de minerales metálicos } \\
\text { Construcción }\end{array}$ & $\begin{array}{l}17,1 \\
16,9 \\
10,3\end{array}$ & $\begin{array}{l}\text { Extracción de minerales metálicos } \\
\text { Agricultura y caza } \\
\text { Establecimientos financieros }\end{array}$ & $\begin{array}{l}67,3 \\
11,0 \\
7,5\end{array}$ & $\begin{array}{l}\text { Calama / } \\
\text { Copiapó } \\
\text { Vallenar } \\
\text { Los Andes } \\
\text { Rancagua-Machalí }\end{array}$ \\
\hline & 2011 & $\begin{array}{l}\text { Extracción de minerales metálicos } \\
\text { Comercio al por menor } \\
\text { Administración pública y defensa }\end{array}$ & $\begin{array}{c}18,3 \\
14,9 \\
9,6\end{array}$ & $\begin{array}{l}\text { Extracción de minerales metálicos } \\
\text { Comercio al por menor } \\
\text { Extracción de otros minerales }\end{array}$ & $\begin{array}{l}62,3 \\
11,1 \\
17,5\end{array}$ & $\begin{array}{l}\text { Calama / Copiapó } \\
\text { Vallenar / Los Andes } \\
\text { Rancagua-Machalí } \\
\text { Antofagasta }\end{array}$ \\
\hline \multirow{2}{*}{ 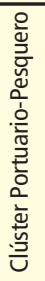 } & 1992 & $\begin{array}{l}\text { Servicios personales y de los hogares } \\
\text { Servicios sociales y otros servicios } \\
\text { comunales conexos } \\
\text { Transporte y almacenamiento }\end{array}$ & $\begin{array}{l}12,3 \\
11,4 \\
8,3\end{array}$ & $\begin{array}{l}\text { Pesca } \\
\text { Minería del carbón } \\
\text { Silvicultura y extracción de madera }\end{array}$ & $\begin{array}{l}75,6 \\
91,9 \\
35,0\end{array}$ & $\begin{array}{l}\text { Iquique-Alto } \\
\text { Hospicio } \\
\text { San Antonio } \\
\text { Gran Concepción } \\
\text { Puerto Montt- } \\
\text { Puerto Varas }\end{array}$ \\
\hline & 2011 & $\begin{array}{l}\text { Comercio al por menor } \\
\text { Pesca } \\
\text { Transporte y almacenamiento }\end{array}$ & $\begin{array}{l}12,5 \\
9,5 \\
9,0\end{array}$ & $\begin{array}{l}\text { Pesca } \\
\text { Agricultura y Caza } \\
\text { Restaurantes y Hoteles }\end{array}$ & $\begin{array}{c}37,5 \\
5,4 \\
4,1\end{array}$ & $\begin{array}{l}\text { Puerto Montt- } \\
\text { Puerto Varas }\end{array}$ \\
\hline \multirow{2}{*}{ 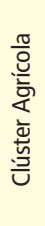 } & 1992 & $\begin{array}{l}\text { Agricultura y caza } \\
\text { Comercio al por menor } \\
\text { Servicios personales y de los hogares }\end{array}$ & $\begin{array}{l}19,6 \\
14,2 \\
12,7\end{array}$ & $\begin{array}{l}\text { Agricultura y caza } \\
\text { Silvicultura y extracción de madera } \\
\text { Fabricación de alimentos, bebidas y tabaco }\end{array}$ & $\begin{array}{l}39,0 \\
25,9 \\
11,4\end{array}$ & $\begin{array}{l}\text { San Felipe / Melipilla } \\
\text { Los Ángeles } \\
\text { Chillán-Chillán Viejo } \\
\text { Osorno }\end{array}$ \\
\hline & 2011 & $\begin{array}{l}\text { Agricultura y caza } \\
\text { Comercio al por menor } \\
\text { Comercio al por mayor }\end{array}$ & $\begin{array}{l}21,6 \\
13,2 \\
11,1\end{array}$ & $\begin{array}{l}\text { Agricultura y caza } \\
\text { Comercio al por mayor } \\
\text { Extracción de otros minerales }\end{array}$ & $\begin{array}{l}13,4 \\
3,3 \\
7,5\end{array}$ & $\begin{array}{l}\text { San Felipe } \\
\text { Melipilla }\end{array}$ \\
\hline \multirow{2}{*}{ 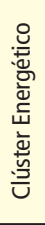 } & 1992 & $\begin{array}{l}\text { Servicios personales y de los hogares } \\
\text { Transporte y almacenamiento } \\
\text { Construcción }\end{array}$ & $\begin{array}{l}14,6 \\
9,8 \\
9,2\end{array}$ & $\begin{array}{l}\text { Servicios personales y de los hogares } \\
\text { Transporte y almacenamiento } \\
\text { Construcción }\end{array}$ & $\begin{array}{l}83,4 \\
2,9 \\
2,5\end{array}$ & $\begin{array}{l}\text { Calama } \\
\text { Copiapó }\end{array}$ \\
\hline & 2011 & $\begin{array}{l}\text { Comercio al por menor } \\
\text { Construcción } \\
\text { Administración pública y defensa }\end{array}$ & $\begin{array}{r}13,9 \\
12,5 \\
10,7\end{array}$ & $\begin{array}{l}\text { Producción de petróleo crudo y gas natural } \\
\text { Minería del carbón } \\
\text { Transporte y almacenamiento }\end{array}$ & $\begin{array}{c}58,1 \\
56,9 \\
1,6\end{array}$ & Punta Arenas \\
\hline \multirow{2}{*}{ 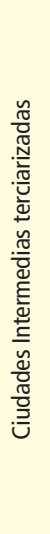 } & 1992 & $\begin{array}{l}\text { Comercio al por menor } \\
\text { Servicios personales y de los hogares } \\
\text { Servicios sociales y otros servicios } \\
\text { comunales conexos }\end{array}$ & $\begin{array}{l}16,9 \\
12,0 \\
11,6\end{array}$ & $\begin{array}{l}\text { Transporte y almacenamiento } \\
\text { Administración pública y defensa } \\
\text { Agricultura y Caza }\end{array}$ & $\begin{array}{l}27,2 \\
27,1 \\
26,5\end{array}$ & $\begin{array}{l}\text { Arica / Antofagasta } \\
\text { La Serena-Coquimbo } \\
\text { Gran Valparaíso } \\
\text { Talca } \\
\text { Temuco-Padre las } \\
\text { Casas } \\
\text { Valdivia / Coyhaique }\end{array}$ \\
\hline & 2011 & $\begin{array}{l}\text { Comercio al por menor } \\
\text { Construcción } \\
\text { Administración pública y defensa }\end{array}$ & $\begin{array}{l}17,6 \\
11,5 \\
8,6\end{array}$ & $\begin{array}{l}\text { Silvicultura y extracción de madera } \\
\text { Agricultura y Caza } \\
\text { Industria de la madera y productos de madera, } \\
\text { incluido muebles }\end{array}$ & $\begin{array}{l}82,3 \\
45,3 \\
53,5\end{array}$ & $\begin{array}{l}\text { Arica } \\
\text { La Serena-Coquimbo } \\
\text { Talca } \\
\text { Temuco-Padre las } \\
\text { Casas } \\
\text { Valdivia / Coyhaique } \\
\text { Iquique-Alto Hospicio } \\
\text { Gran Concepción } \\
\text { Chillán-Chillán Viejo } \\
\text { Osorno } \\
\text { San Antonio } \\
\text { Los Ángeles }\end{array}$ \\
\hline
\end{tabular}


pierden su condición de "especializadas" en ciertas actividades primarias, como la pesca y la agricultura. La especialización parece quedar reservada para las actividades primarias orientadas fundamentalmente a la exportación, como lo demuestra la estabilidad del clúster minero y la permanencia de Puerto Montt-Puerto Varas en el clúster portuariopesquero, lo que se explica por la relevancia local de la industria del salmón.

De esta forma, las ciudades que no logran entran en el circuito de la exportación de recursos naturales a gran escala, se caracterizan cada vez más por una economía local terciarizada, donde además el sector inmobiliario y de la construcción, el comercio adquiere un rol preponderante. Este tipo de actividad se transforma muchas veces en el principal dinamizador de las economías locales, reemplazando progresivamente el rol que en algún momento tuvieron las actividades primarias en estas ciudades.

Sin embargo, teniendo en cuenta esta tendencia general hacia la homogenización de los mercados laborales de algunas ciudades, o bien, la diferenciación respecto a otras ciudades que se mantienen altamente especializadas en ciertas actividades primarias, es relevante preguntarse cuáles son los factores que explican esta homogeneidad y en qué ámbitos se mantiene algún grado de diferenciación entre las ciudades intermedias terciarizadas. Además, si esta tendencia de urbanización de la base económica y del mercado laboral genera o no mayor vulnerabilidad socio-territorial, en la medida en que las ciudades se vuelven dependientes de un sector fuertemente sensible al ciclo económico.

Además, en un escenario en el que la relación entre crecimiento urbano y homogenización del mercado del trabajo parece ocurrir en buena parte de los cluster encontrados. Los desafíos de esta transformación tiene que ver con las características del empleo que se va generando en las ciudades que transitan hacia esta estructura, ya que a diferencia del sector primario o secundario, parecen ser empleos de baja calidad. Por otro lado, ante el aumento de población y crecimiento urbano, tanto en expansión como en densificación, el desafío consiste en evitar las consecuencias no deseadas del proceso de modernización urbana, como la segregación residencial y la fragmentación urbana. Si bien no es posible constatar un relación directa entre crecimiento urbano, transformación del mercado laboral y consecuencias sociales negativas, el análisis realizado permite detectar un proceso de transformación estructural, en una buena muestra de ciudades en Chile en diferentes escalas, que adelantan una transformación socio-territorial, como ha ocurrido en los casos consolidados de metropolitzación (De Mattos et al., 2005).

Por lo tanto, el tránsito hacia una dinámica eminentemente urbana en el mercado del trabajo en las principales ciudades en Chile, debe ser observado cuidadosamente, con el objetivo de construir ciudades más sustentables en el contexto de una economía financierizada y un proceso de urbanización planetaria. 


\section{Luis Fuentes}

Pontificia Universidad Católica de Chile, Instituto de Estudios Urbanos y Territoriales. Santiago, Chile. Ifuentes@uc.cl

\section{Felipe Link}

Pontificia Universidad Católica de Chile, Instituto de Estudios Urbanos y Territoriales. Santiago, Chile. felipe.link@uc.cl

\section{Felipe Valenzuela}

Pontificia Universidad Católica de Chile, Instituto de Estudios Urbanos y Territoriales. Santiago, Chile. felipevalenzuelao@gmail.com

\section{Nota}

(*) Este trabajo fue realizado gracias al apoyo del proyecto Fondecyt $n^{\circ} 1141157$ y de los proyectos Fondap Cedeus no 15110020 y Fondap Coes no 15130009, de la Comisión Nacional de Investigación Científica y Tecnológica Conicyt.

\section{Referências}

ANTUNES, R. (1995). ¿Adiós al trabajo? Ensayo sobre la metamorfosis y centralidad del mundo del trabajo. São Paulo, Cortez.

AQUEVEDO, E. (1997). América Latina, de la substitución de importaciones al auge neoliberal. Revista Ultima Década n. 7, sectores populares y acción colectiva. Disponible en: http://www.cidpa.org/ decada7.asp.

BRENNER, N. (2013). Tesis sobre la urbanización planetaria. Nueva Sociedad, n. 243, pp. 38-66. Concurso Nacional de Proyectos Fondecyt Regular.

BUZAI, G. (2003). Mapas sociales urbanos. Buenos Aires, Lugar.

CÁRDENAS, A.; LINK, F. y STILLERMAN, J. (2010). Qué significa el trabajo hoy. Cambios y continuidades en una sociedad global. Santiago, Catalonia.

CENTRO DE ESTUDIOS PÚBLICOS (1992). EI Ladrillo: bases de la política económica del gobierno militar chileno. Santiago, CEP.

CHESNAIS, F. (2003). La teoría del régimen de acumulación financiarizado: contenido, alcance e interrogantes. Revista de Economía Crítica, n. 1, pp. 37-72.

DAHER, A. (1995). Efecto concentrador de los fondos inmobiliarios. Revista de Ciencias Sociales. San José de Costa Rica, n. 68, pp. 17-32. 
DE MATTOS, C. (2016). Financiarizacion, mercantilización y metamorfosis planetaria: lo urbano en valorización del capital. Sociologías. Porto Alegre, v. 18, n. 42, pp. 24-52.

DE MATTOS, C.; FUENTES, L. y LINK, F. (2016). “Mutations in the Latin American metrópolis: Santiago de Chile under neoliberal dynamics". In: NEL-LO, O. y MELE, R. (eds.). Cities in the 21st Century. Routledge/Enel Foundation.

DE MATTOS, C. y RIFFO, L. (2005). “Globalización, redes, nodos y dinámica metropolitana: El Gran Santiago en los noventa". In: DE MATTOS, C.; FIGUEROA, O.; JIMÉNEZ, R.; ORELLANA, A. y YAÑEZ, G. (eds.). Gobernanza, competitividad y redes: La gestión en las ciudades del siglo XXI . Santiago, Colección Rideal-Eure Libros.

DÍAZ, X.; GODOY, L.; STECHER, A. y TORO, J. P. (eds.) (2006). Trabajo, Identidad y vinculo social: reflexiones y experiencias en el capitalismo flexible. Santiago de Chile, Centro de Estudios de la Mujer y Universidad Diego Portales.

DIVISIÓN ESTADÍSTICA DE NACIONES UNIDAS (s.d.). CIIU Rev.3. Estructura detallada y notas explicativas. Disponible en: http://unstats.un.org/unsd/cr/registry/regcst.asp?Cl=2\&Lg=3. Recuperado el: 31 enero 2017

FERRADA, L. M. y ZARZOSA, P. (2010). Diferencias regionales en la participación laboral femenina en Chile. Cuadernos de Economía, v. 47, n. 136, pp. 249-272.

GÁRATE CHATEAU, M. (2012). La revolución capitalista de Chile. Santiago, Ediciones Universidad Alberto Hurtado.

HARVEY, D. (2010). El enigma del capital. Madrid, Akal.

HIDALGO, R.; DE MATTOS, C. e ARENAS, F. (2009). Chile: del país urbano al país metropolitano. Santiago, Pontifícia Universidad Católica de Chile.

KAZTMAN, R. (2001). Seducidos y abandonados: el aislamiento social de los pobres urbanos. Revista de la Cepal n. 75. Santiago.

KAZTMAN, R. Y RIBEIRO, L. (2008). Metrópolis y Sociabilidad: reflexiones sobre los impactos de las transformaciones socioterritoriales de las grandes ciudades en la cohesión social de los países de América Latina. Documento de trabajo Observatorio das Metropoles, Universidad Federal de Río de Janeiro, Brasil.

LEFEBVRE, H. (1970). La révolution urbaine. Paris, Gallimard.

LEÓN, A. y MARTÍNEZ, J. (2007). "La estratificación social en Chile hacia fines del siglo XX”. In: FRANCO, R; LEÓN, A. e ATRIA, R. Estratificación y movilidad social en América Latina. Transformaciones estructurales de un cuarto de siglo. Santiago, LOM.

LINK, F. (2008). Fragmentación urbana y consecuencias sociales. Revista Ciudades n. 77. México, RNIU. (2010). Globalización, vulnerabilidad y riesgo en la nueva configuración socio-territorial del trabajo en el Area Metropolitana de Santiago. Tese de Doutorado. Santiago, Pontificia Universidad Católica de Chile.

LUFÍN VARAS, M. e ATIENZA ÚBEDA, M. (2010). Diferencias entre la composición sectorial y ocupacional de las principales ciudades chilenas. Eure. Santiago, v. 36, n. 108, pp. 75-93.

MOULIAN, T. (1997). Chile: anatomía de un mito. Santiago, Lom. 
OIT (1987). CIUO: Clasificación Internacional Uniforme de Ocupaciones. Rescatado de: http://www.ilo. org/public/spanish/bureau/stat/isco/isco88/major.htm (1998). Chile: Crecimiento, empleo y el desafío de la justicia social. Santiago de Chile, OIT.

PREVOT-SHAPIRA, M. (2001). Fragmentación social y espacial. Conceptos y realidades. Revista Perfiles Latinoamericanos n. 19. México.

RIFFO, L. (2003). Globalización, Metropolización y Mercados de Trabajo. Revista América Latina. Santiago, Universidad Arcis.

ROJAS, S. (1984). Políticas de erradicación y radicación de campamentos: 1982-1984, discursos, logros y problemas. Programa FLACSO, n. 215.

RUBIO, R. (2002). El estudio geográfico de los mercados del trabajo metropolitanos: enfoques teóricos y consideraciones metodológicas. Revista de Geografía Norte Grande, n. 29. Santiago.

RUIZ-TAGLE, J. (1985). El Sindicalismo chileno después del Plan Laboral. Santiago, Programa de Economía del Trabajo.

(2000). Exclusión social en el Mercado de trabajo en Mercosur y Chile. Santiago, OIT-Fundación Ford.

SABATINI, F. (2000). Reforma de los mercados de suelo en Santiago, Chile: efectos sobre los precios de la tierra y la segregación residencial. Eure. Santiago, v. 26, n. 77, pp. 49-80.

SASSEN, S. (2001). Los espectros de la globalización. Buenos Aires, FCE.

TORCHE, F. Y WORMALD, G. (2007). “Chile, entre la adscripción y el logro”. In: FRANCO, R.; LEÓN, A. e ATRIA, R. Estratificación y movilidad social en América Latina. Transformaciones estructurales de un cuarto de siglo. Santiago, Lom.

WELLER, J. (2011). Panorama de las condiciones de trabajo en América Latina. Revista Nueva Sociedad, n. 232, pp. 32-49.

WORMALD, G. y RUIZ-TAGLE, J. (1999). Exclusión social en el mercado del trabajo: el caso de Chile. Documentos de Trabajo, n. 106, Oficina Internacional del Trabajo/Fundación Ford. Santiago de Chile, OIT-Fundación Ford.

Texto recebido em 26/set/2016

Texto aprovado em 2/dez/2016 
